\title{
Preliminary Experience and Extended Applications for the Use of Autologous Platelet-Rich Plasma in Hair Transplantation Surgery
}

\author{
Joseph Greco, PhD, PA/C, Robert J. Brandt Siesta Key, Florida
}

Joe Greco has indicated no financial interest relevant to this article; Robert Brandt is president of Blood Recovery Systems.

The use of platelet rich plasma (PRP) in hair restoration surgery reported increased yield when utilized as a graft storage medium (Uebel, 2005). ' When grafts are bathed in activated PRP, there appears to be higher graft survival and quicker healing.

Based on five months of experience involving more than 30 cases, the authors suggest expanding the use of PRP in hair restoration surgery for the following reasons: (1) to enhance donor site wound healing, (2) to decrease the incidence of infection, (3) to reduce donor scarring, (4) to increase donor scar tensile strength, (5) to enhance recipient site healing, and (6) to be utilized as an effective treatment protocol in severe cases of wound dehiscence or infection. In addition to the PRP, platelet poor plasma (PPP) also has potent sealant properties that can be utilized for hemostasis during the procedure.

Platelet-derived growth factor (PDGF) is the evolutionary sentinel growth factor that initiates all wound healing. Platelet rich plasma (PRP) contains several growth factors, including PDGF and transforming growth factor-beta (TGF-beta 1) at high levels and vascular endothelial growth factor (VEGF).

PDGF's main function is to stimulate cell replication (mitogenesis) of healing capable stem cells. It also stimulates cell replication of endothelial cells. This will cause budding of new capillaries into the wound (angiogenesis), a fundamental part of all wound healing. In addition, PDGF seems to promote the migration of perivascular healing capable cells into a wound and to modulate the effects of other growth factors. Numerous studies and practical applications have also demonstrated how growth factors are essential for regulating the cellular events involved in wound healing by attracting cells to the wound, stimulating proliferation, and significantly influencing matrix deposition (Declair, 1999). ${ }^{2}$

TGF-beta is extremely important because it affects most aspects of tissue wound repair, namely initiation and termination, and also promotes differentiation and proliferation (Choi and Fuchs, 1990). ${ }^{3}$ PDGF improves dermal regeneration, acts locally to promote protein and collagen synthesis, causes endothelial migration or angiogenesis (Ross, 1987), ${ }^{4}$ and induces the expression of TGF-beta (Pierce, et al., 1989). ${ }^{5}$

It was further established that wounds treated with PRP gel exhibited not only enhanced wound repair compared to control, but possess more organized collagen than control tissues, without excessive deposition of connective tissue or scar formation (Carter, et al., 2002). ${ }^{6}$ This equine study by Carter, et al. demonstrated biopsy wounds treated with PRP gel to be densely organized, tightly packed fiber bundles parallel to the overlying epidermis suggesting the dense collagen lattice had increased tensile strength in the repaired wound.

The use of PDGF in surgery is widely documented and has become standard intra-operative and post-operative protocol to promote hemostasis, accelerate wound healing, and decrease the incidence of wound infection.

It is therefore suggested that PRP gel is an excellent proto$\mathrm{col}$ in hair transplantation for donor wound closures. Preoperatively, 50cc of blood is drawn from the patient and processed according to the established protocol to create the PRP gel. After the donor strip is harvested, the subcuticular layer is closed with 3.0 Monocryl, activated PRP gel is injected into the wound from end to end (Figure 1), and the second layer is approximated with a running 3.0 Prolene suture. After utilizing PRP gel in the donor site, wounds appear to bleed less post-operatively than those not treated with PRP.

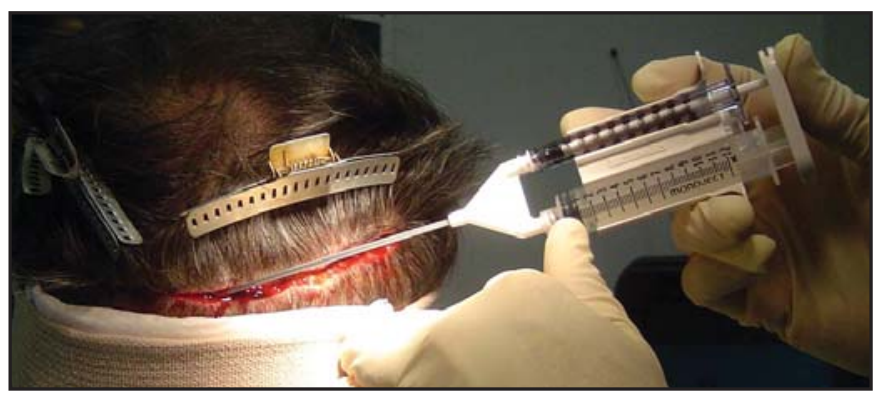

Figure 1. Gel injected into the wound, from end to end.

Fear of linear donor scaring is a major concern in our patients today. In our experience, the use of platelet-rich plasma during donor closure results in better healing and less scarring.

After the follicular units are dissected, they are bathed in activated PRP gel (PRP can be activated with calcium chloride/thrombin or fibrinogen and becomes a gel-like substance) approximately 15 minutes prior to implantation (Figure 2).

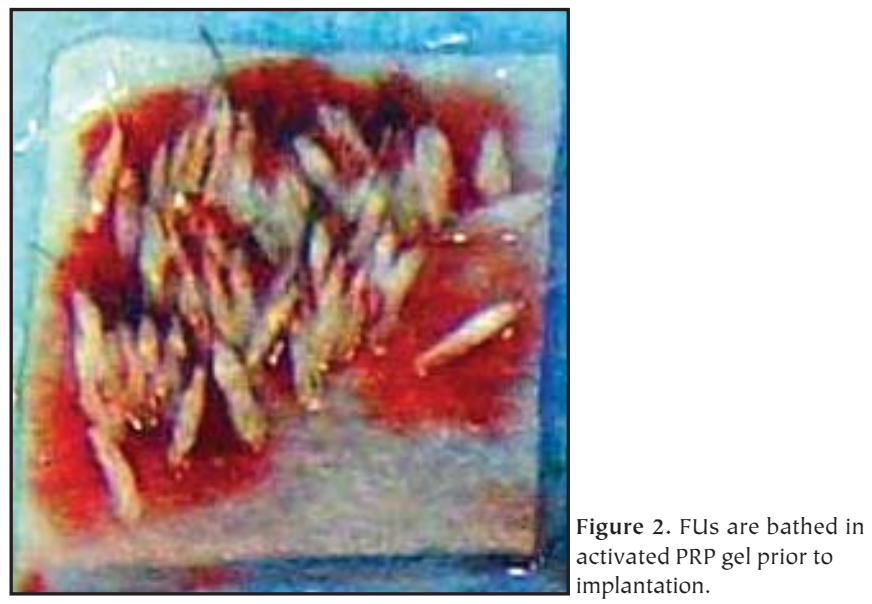

While dissection is ongoing and the graft design pattern is completed, the PRP is then injected into the recipient scalp area after the graft pattern is completed to maximize the multiple effects of growth factors. The PRP provides an en- 


\section{Platelet-Rich Plasma}

continued from page 131

riched environment of concentrated growth factors to accelerate the wound response, thus promoting healing and angiogenesis for the newly implanted follicular units (Figure 3 ).

Injecting PRP into the recipient area may have other advantages for the non-transplanted hairs because PRP contains several growth factors, including PDGF and VEGF. Takakura, et al. $(1996)^{7}$ demonstrated that PDGF signals are involved in both epidermis-follicle interaction and the dermal mesenchyme interaction required for hair canal formation and the growth of dermal mesenchyme, respectively. In 2001, Yano, et al. ${ }^{8}$ identified VEGF as a major mediator of hair follicle growth and cycling providing the first direct evidence that the improved follicle vascularization promotes hair growth and increases follicle and hair size.

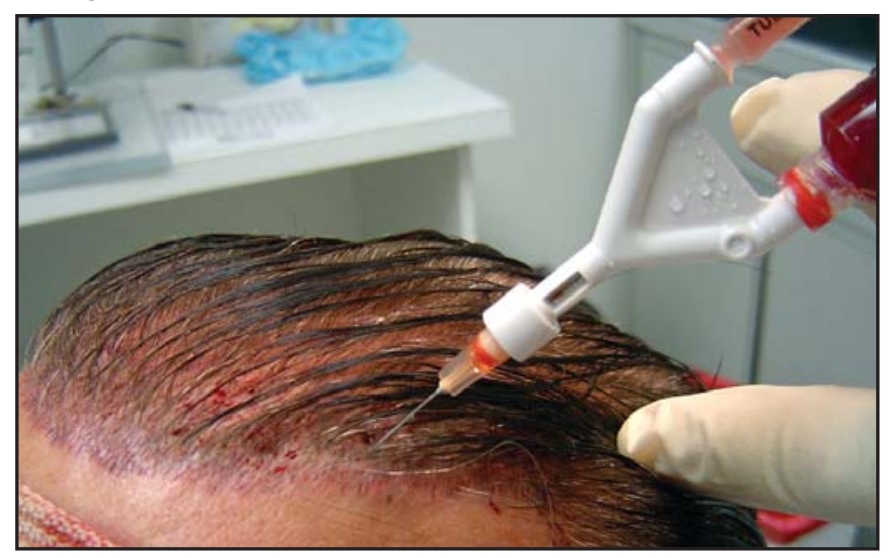

Figure 3. PRP promotes healing and angiogenesis in newly implanted FUs.

This author has observed a more rapid healing after injecting PRP into the recipient site in hair transplantation. Based on the previously mentioned studies regarding the effects growth factors have on hair growth, studies are planned to test the effects PRP and growth factors have on the nontransplanted hair.

In 2003, one of us demonstrated rapid healing and hair regrowth utilizing PRP on a severely traumatized wound in an equine model. While it generally takes nine months for a wound such as this to heal, if the animal survives at all, in this PRP-treated animal, rapid healing of the wound occurred. At one month, complete wound closure and hair regrowth was evident, which never occurs in these cases. Enlarged photos of this case can be seen at the website http:// bloodrecovery.com/wound_ba2.htm. ${ }^{9}$
This equine case is a significant example of the extraordinary effects that PRP has on rapid wound repair and hair regrowth in especially difficult cases. It illustrates yet another very valuable use for PRP, especially, in cases of severe infection or wound dehiscence. Rapid use of PRP in this instance cannot only promote healing of the infected wound, but will also promote the regrowth of hair, thus avoiding possible impending scarring traumatic alopecia.

In conclusion, we are seeing encouraging results with these expanded applications for PRP. Further experience will help delineate the role for this exciting technology in our specialty.

\section{REFERENCES}

1. Uebel, C. O. Presented at the Annual Scientific Meeting of the American Society of Plastic Surgeons in Philadelphia, Pennsylvania, Oct. 9-13, 2004.

2. Declair, V. (1999). The importance of growth factors in wound healing. Ostomy Wound Manage. 45; 64-68.

3. Choi, Y., Fuchs, E. (1990). TGF-beta and retnoic acid regulation of growth and modifiers of differentiation human epidermal cells. Crell regal. 1; 791-809

4. Ross, R. (1986). Platelet-derived growth factor. Am. Rev. Med. 38; 71-79.

5. Pierce, G. F., et al. (1989). Transforming growth factor B reverses the glucocorticoid-induced wound healing defect in rats: possible regulation in microphages by plateletderived growth factor. Proc. Natl. Acad. Sci. 86; 2229-233.

6. Carter, C. A., et al. (2003). Platelet-rich plasma gel promotes differentiation and regeneration during equine wound healing. Experimental and Molecular Pathology 74; 244-55.

7. Takakura, N., et al. (1996). Involvement of platelet-derived growth factor receptor-a in hair canal formation. Journal of Investigative Dermatology 107; 770-77.

8. Yano, K., Brown, L., and Detmar, M. (February 2001). Control of hair growth and follicle size by VEGF-mediated angiogenesis. J Clin Invest 107(4); 409-1 7.

9. Brandt, R. (2003). Internet website posting. http:// bloodrecovery.com/wound_ba2.htm

Editor's note: This preliminary information takes the work of Uebel one step further, suggesting that PRP may be of use not only for "basting" grafts but also when injected into recipient sites and in the donor wound. It will be interesting to see if this anecdotal data can be repeated by others and followed with convincing clinical studies. - IC
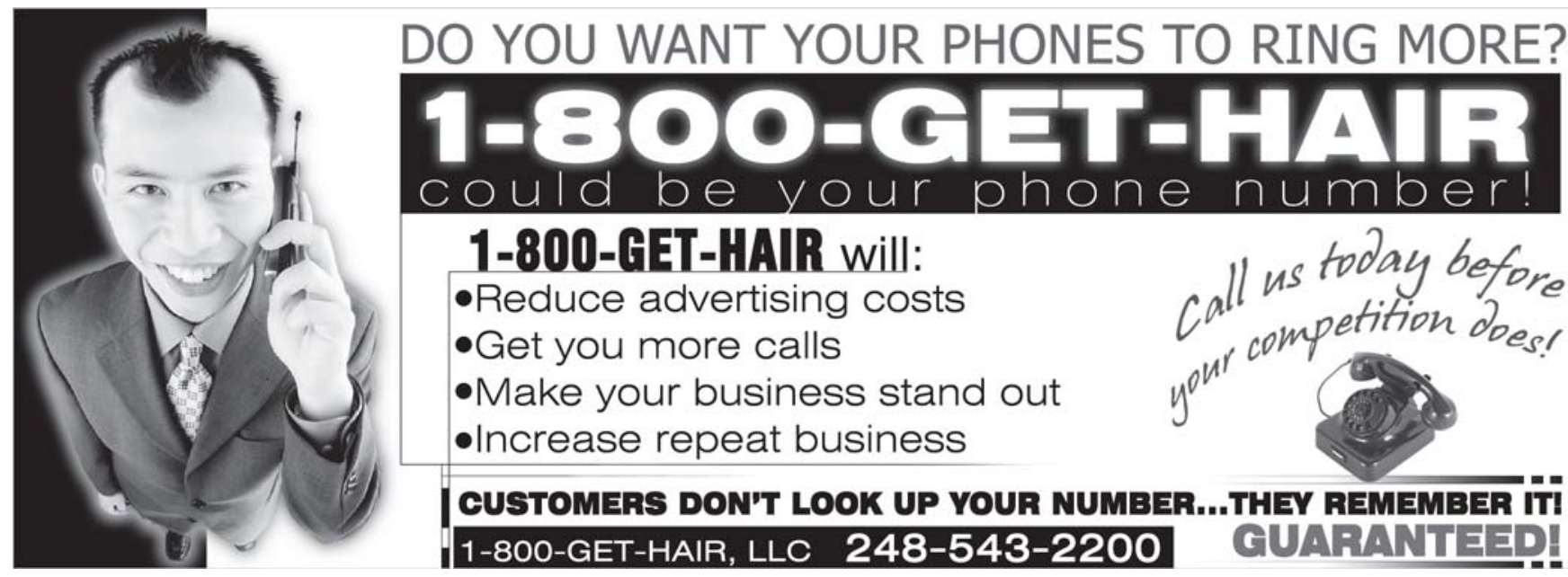